\title{
Monte Carlo Calculation of linear attenuation coefficients and photon scattering properties of novel concretes loaded with Osmium, Iridium and Barite nanoparticles
}

\author{
Samira Keramat JOU a, Asghar MESBAHI ${ }^{a,{ }^{*}}$, Reza Eghdam ZAMIRI ${ }^{\mathrm{b}}$, Farshad SEYEDNEJADa \\ ${ }^{a}$ Medical Radiation Sciences Research Team, Tabriz University of Medical Sciences, Tabriz, Iran \\ ${ }^{b}$ Radio-Oncology Department, Shahid Madani Hospital, Tabriz, Iran \\ ${ }^{*}$ E-mail address: amesbahi2010@gmail.com
}

\begin{abstract}
Introduction: Recent studies have shown that the use of high-density nanoparticles (NPs) in concrete composition improves its radiation shielding properties. In the present study, the linear attenuation coefficients and photon scattering properties of newly developed high-density Nano-concretes have been calculated using the MCNPX Monte Carlo code. Material and methods: The shielding properties of Nano-concretes containing 10\%, 20\%, and 30\% weight percentage of Osmium, Iridium and Barite NPs (100 nm) as well as ordinary concrete were investigated. The 6 and $18 \mathrm{MV}$ photon beams of Varian Linac and ${ }^{60} \mathrm{Co}$ photons were used for simulation. Photon scattering flux was calculated for all Nanoconcretes with $30 \mathrm{wt} \%$ of NPs and ordinary concrete at different angles.

Results: In general, by adding Iridium, Osmium and Barite NPs to ordinary concrete, the linear attenuation coefficients increased. Despite a lower density relative to Iridium and Osmium, Nano-concretes containing Barite exhibited a higher linear attenuation coefficient due to their higher electron density.

Conclusions: The results revealed a dependence between the scattered photon flux and the effective atomic number of Nano-concretes. With increasing the atomic number of fillers, the intensity of the scattered photon flux enlarged. Also, the scattered flux was higher for all types of concretes at 180 degrees relative to other angles.
\end{abstract}

Key words: osmium; iridium; composite shield; nanoparticles; linear attenuation coefficient; MCNPX.

\section{Introduction}

Linear accelerators (Linacs) are one of the most common devices used in radiotherapy that produce high-energy photons and electrons in the megavoltage (MV) energy range. Due to the increasing use of Linacs for the treatment of cancer patients, the radio-protection of staff and patients against the harmful effects of these ionizing radiations has become a crucial subject. ${ }^{1}$

Concretes are applied conventionally as both shielding and construction materials to build a radiotherapy bunker. The typical concrete composition includes: Portland cement, water, sand, and aggregates such as rock and sand. To boost the physical and mechanical properties as well as radiation protection, high-density materials such as Barite, Galena, and Magnetite are added to ordinary concrete. ${ }^{2}$

These concretes are called heavy or high-density concretes (HDCs) and play an important role in the construction of bunkers with augmented protection against ionizing radiation and increased available space. ${ }^{3}$ Photons and neutrons are the main types of radiation in linear accelerators that must be attenuated and stopped by a suitable shielding material in the walls and ceilings of radiotherapy bunkers. A large number of experimental and theoretical studies on the HDCs have been performed to introduce the best shielding material with the highest mass attenuation coefficient. There are several methods for designing bunkers for radiotherapy centers, including methods based on simplified equations and Monte Carlo (MC) methods. ${ }^{4}$ In this regard, experimental investigations require a lot of time and are very costly. Researchers have employed MC methods to overcome these limitations. By using MC simulation, it is easy to examine changes in radiotherapy bunkers' various parameters, such as dimensions and maze geometry. The MC simulation also makes it possible to examine parameters that are not measurable practically. For example, due to the limitations and difficulties associated with the dosimetry of neutrons in environments with mixed beams, including photons and neutrons, MC simulation has shown itself as a suitable method for calculating photo-neutron contamination. ${ }^{5}$ For instance, in the study by Juste et al., neutron dose values in different parts of the bunker were calculated once using MC method, and once practically, using the dosimeter. The results of both methods were significantly consistent, which confirms the capability of the MC method. ${ }^{6}$ 
The atomic number and density of the elements used in the concrete composition affect the attenuation of high-energy photons. ${ }^{7}$ Studies have shown that adding high-density materials such as Barite, Galena, Colemanite, Lead-oxide and perlite minerals to the concrete mixture improved the linear attenuation coefficient $(\mu)$ and decreases half-value layer (HVL) and mean free path (MFP) values. ${ }^{8-11}$

The use of nanoparticles (NPs) in concrete composition increases the surface-to-volume ratio, diminishes fine concrete pores and improves the compressive strength and physical strength of concrete. ${ }^{12}$ In previous studies, comparisons of microparticles- and NPs-containing concretes have shown higher attenuation coefficients and lower HVLs to protect against energetic photons with Nano-concretes (NCs). ${ }^{\mathbf{1 3 - 1 5}}$

The scattering properties of photons impinging on the walls inside the radiotherapy bunker affect the patients' received whole-body dose from neutrons and photons. In addition, the dose of photons and neutrons reaching the entrance door of the maze is significantly impacted by the scattering properties of the concrete walls. Studies have reported that $75 \%$ of the neutrons at the entrance door of the maze are scattered neutrons. ${ }^{\mathbf{1 6 , 1 7}}$ Studies have also publicized that the intensity of photons and neutrons scattered at any angle by different radiation shielding materials and concretes depends on the composition of the concrete and shielding materials used.

For example, Mesbahi et al. have shown that the scattering of photo-neutrons by different HDCs significantly depends on their composition. The use of high-density elements such as Iron and Lead increases the production of photo-neutrons in the walls. ${ }^{18}$ The effect of different commercially available concretes on photo-neutron doses in the isocenter and entrance door of the maze revealed that the use of high-density concretes, in order to use more space in the radiotherapy bunker or other purposes, enhanced the neutron dose reached to the entrance door of the maze. ${ }^{19}$ It was reported that the number of neutrons at the entrance door of the maze varied up to $36 \%$, depending on the atomic composition of the concrete. In another study, evaluating the equivalent neutron dose produced by photo-neutrons inside primary barriers revealed that primary barrier shielding with a metal sheet for high energy photons, proper neutron shielding material should be constructed to reduce photo-neutron dose. ${ }^{\mathbf{2 0}}$ In an MC study, the results showed that adding $5 \mathrm{wt} \%$ and 10 wt\% Colemanite to Barite-concrete reduced photo-neutron production for $18 \mathrm{MV}$ photons. ${ }^{21}$ Furthermore, it has been reported that the scattering intensities of photons and neutrons vary depending on the composition of the HDC. ${ }^{\mathbf{2 2}}$

In the current study, the addition of two high-density NPs including Osmium (Os, $\mathrm{Z}=76)$ and Iridium $(\mathrm{Ir}, \mathrm{Z}=77)$ to the ordinary concrete was considered. Osmium is the densest element in nature with a density of $22.59 \mathrm{~g} / \mathrm{cm}^{3}$. Its most abundant stable isotope is $\mathrm{Os}^{190}$ with 114 neutrons. Therefore, Osmium with the highest density in nature could be a good choice as a protective substance in radiation therapy bunkers. Iridium is the second densest metal after Osmium, with a density of $22.56 \mathrm{~g} / \mathrm{cm}^{3}$. Iridium is highly resistant to corrosion and erosion even at temperatures up to $2000^{\circ} \mathrm{C}$. However, its high melting point, hardness, and brittleness make it very difficult to shape the Iridium, so its powder is used. For the first time, these elements in the form of NPs were added to ordinary concrete and their resultant properties were compared to the Barite (Ba)concrete. All of these compounds are modelled as NPs using MC simulation. Linear attenuation coefficient and scattering properties of these NCs were calculated for the 6 and $18 \mathrm{MV}$ photon beam from Varian Linac and ${ }^{60} \mathrm{Co}$ gamma rays $(1.25$ $\mathrm{MeV})$.

\section{Method and Materials}

\section{Simulation of NPs and NCs}

In this study, MCNPX MC code (version 2.7.0, Los Alamos National Laboratory cross-section libraries data) has been used for all simulations. ${ }^{23}$ The Nano-sized Ir, Os, and Ba particles with a radius of $50 \mathrm{~nm}$ have been defined into ordinary concrete by using Lattice (LAT) and Universe (u) properties of MCNPX in the input file. The concrete matrix was filled in three weight percentages (wt $\%$ ) of $10 \%, 20 \%$, and $30 \%$ of mentioned NPs. After obtaining the required number of NPs in each composite, the dimensions of a cubic cell that surrounds a single particle were calculated. The schematic view of the nanoparticles added matrix can be seen in Figure 1.

The NC shield is located between the radiation source and the detector at a distance of $100 \mathrm{~cm}$ from each of them. The narrow beam geometry for the photon was simulated using a disk source inside a Lead cylinder to provide a parallel emitting source with a diameter of $10 \mathrm{~mm}$. The detector was simulated as a small cylinder in a larger Lead cylinder, as shown in Figure 2.

\section{Validation of MC geometry}

In order to evaluate the accuracy and validity of the developed MC code, we modelled pure Lead and ordinary concrete as an attenuator by considering elemental density properties. Then obtained results have been compared with standard WinXCOM data. The calculated $\mu$ values by MCNPX agreed well with $\mathrm{XCOM}$ results. Thus, a validated input code has been used for the calculation of the radiation linear attenuation coefficients of concrete shields doped by different percentages of NPs.

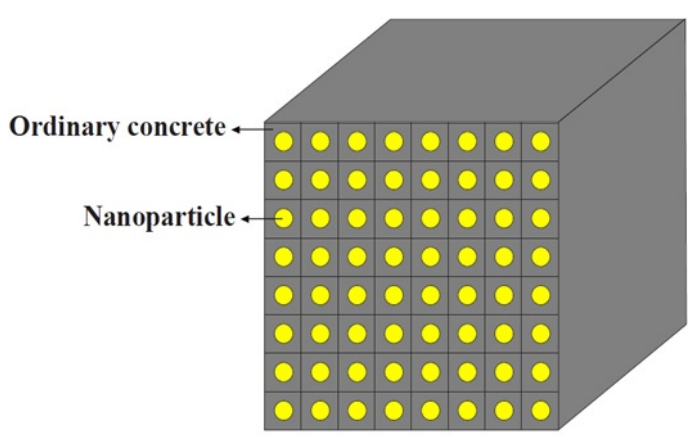

Figure 1. Geometry of Nano-concrete, filling with nanoparticles with Lattice and Universe cards in MCNPX Monte Carlo code. 


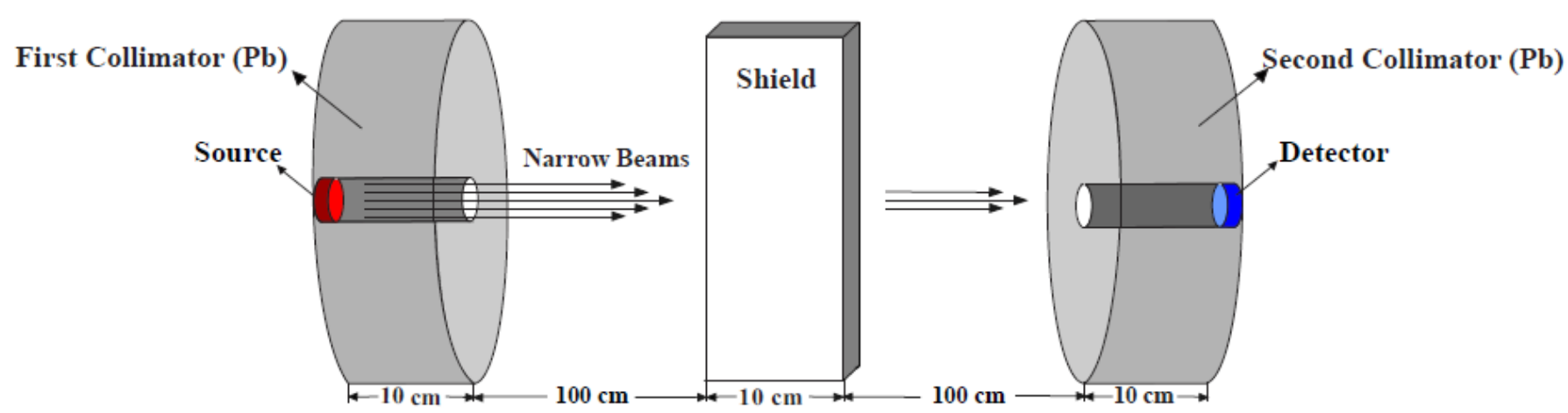

Figure 2. Simulation geometry employed by the MCNPX Monte Carlo code in this study for calculation of linear attenuation coefficient.
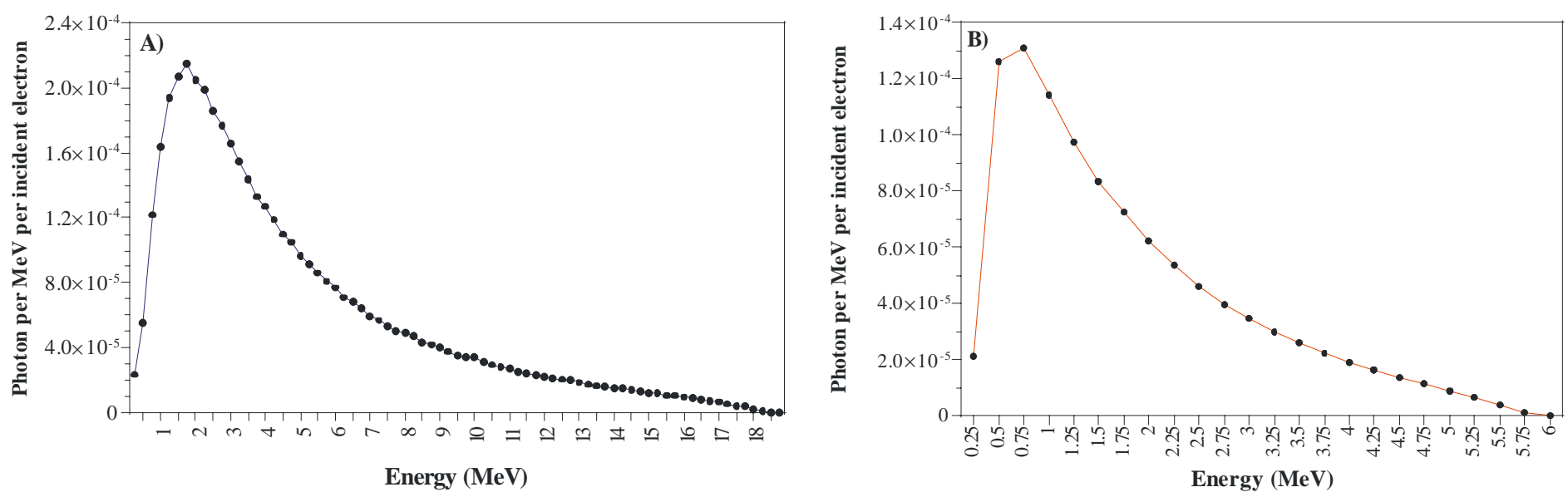

Figure 3. The photon energy of A) $18 \mathrm{MV}$ and B) $6 \mathrm{MV}$ spectra used for Monte Carlo calculations derived from Sheykh-Bagheri et al.

\section{Measurements of $\boldsymbol{\gamma}$-ray transmission}

The linear attenuation coefficient of samples was obtained by the Beer-Lambert equation:

$I=I_{0} e^{-\mu x}$

Eq. 1

In this formula, $I_{0}$ is the number of photons counted in the nonabsorbent state, and $I$ represents the number of photons that have passed thickness of $x$ through of the shield. Also, $\mu$ indicates the amount of linear attenuation coefficient. To obtain $\mu$ amount of each sample, six different thicknesses of NC, including 5, 10, $20,30,40$, and $50 \mathrm{~mm}$ were located between radiation source and detector. The beam transmission was recorded by $\mathrm{MC}$ simulation. Using the obtained data, a transmission-thickness diagram was drawn. In this diagram, the vertical axis showed the value of $\ln I_{0} / I$, which was equivalent to the intensity of transmission photons through the thickness of the shield, and the horizontal axis indicated the thickness of the shield. The slope of the obtained line was the linear attenuation coefficient for each sample. The $\mu$ values obtained for NCs containing Ir, Os, and $\mathrm{Ba}$ were compared with the $\mu$ values of ordinary concrete for all three photon energies of $6 \mathrm{MV}, 18 \mathrm{MV}$ and ${ }^{60} \mathrm{Co}(1.25$ $\mathrm{MeV}$ ) photons. For the 6 and $18 \mathrm{MV}$ photon beams, the photon beam spectrum of the Varian Linac reported by Sheikh Bagheri et al have been used as the photon source (Figure 3). ${ }^{\mathbf{2 4}}$

\section{Assessment of scattered photon flux}

For calculating the flux of scattered photons, an NC shield, containing $30 \mathrm{wt} \% \mathrm{Ir}$, Os, and Ba NPs with a thickness of 250 $\mathrm{cm}$, was placed at a distance of $330 \mathrm{~cm}$ from the photon source, which is modelled as a disk plate with a diameter of $10 \mathrm{~cm}$. Ten spherical cells, with a diameter of $10 \mathrm{~cm}$ were located at a distance of $340 \mathrm{~cm}$ from the concrete shield at angles of 90, 100, $110,120,130,140,150,160,170$, and 180 degrees to the incident beam (Figure 4). The NC matrix was modelled to calculate the photon scattering flux with different angles. The F4 tally was used to calculate the flux of scattered photons from each detector cell per initial photon $\left(n / \mathrm{cm}^{3}\right)$. In these simulations, the scattered photon flux of the $\mathrm{NC}$ at different angles was calculated for all samples of NCs and compared with ordinary concrete. 


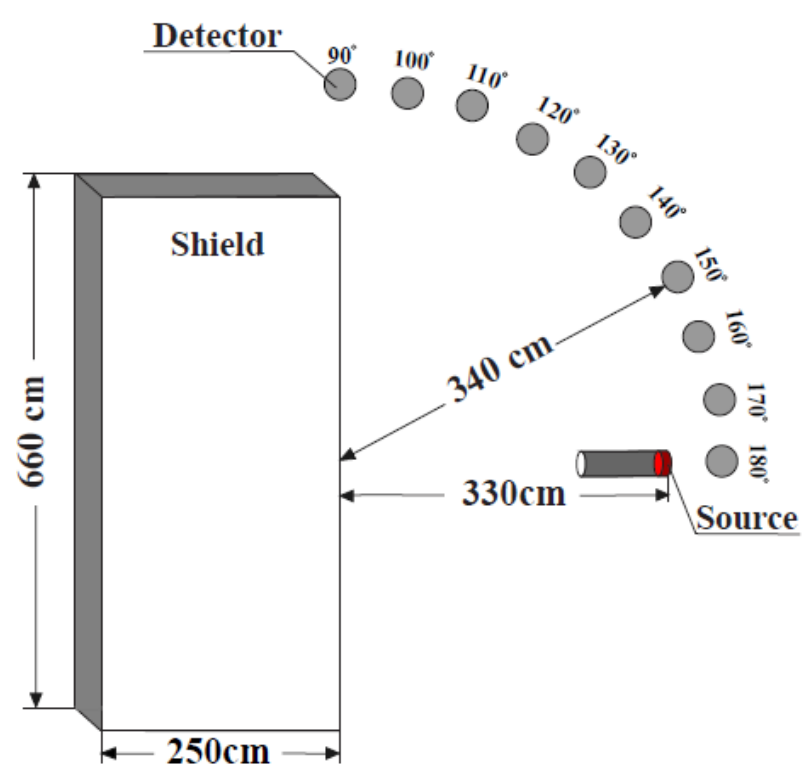

Figure 4. Simulation geometry employed by the MCNPX Monte Carlo code for calculation of photon scattering from a Nanoconcrete shield.

Table 1. Benchmarking of Monte Carlo model against the available data on Lead and ordinary concrete from WinXCOM database.

\begin{tabular}{ccccc}
\hline \hline $\begin{array}{c}\text { Energy } \\
(\mathbf{M V})\end{array}$ & $\begin{array}{c}\text { Density } \\
\left(\mathbf{g} / \mathbf{c m}^{\mathbf{3}}\right)\end{array}$ & $\begin{array}{c}\text { Developed } \\
\text { MCNPX }\end{array}$ & WinXCOM & $\begin{array}{c}\text { Differences } \\
\text { \% }\end{array}$ \\
\hline \multirow{2}{*}{1} & Concrete (2.3) & 0.1466 & 0.14743 & $0.56 \%$ \\
& Lead (11.34) & 0.7915 & 0.80514 & $1.7 \%$ \\
\multirow{2}{*}{6} & Concrete (2.3) & 0.0618 & 0.06187 & $0.1 \%$ \\
& Lead (11.34) & 0.4894 & 0.49794 & $1.7 \%$ \\
\multirow{2}{*}{18} & Concrete (2.3) & 0.047 & 0.04784 & $1.8 \%$ \\
& Lead (11.34) & 0.6926 & 0.6804 & $1.8 \%$ \\
\hline \hline
\end{tabular}

\section{Results}

Here, the photon shielding properties of 3 types of heavy NCs have been calculated. In the first step, the validity of the MC model for calculating $\mu$ value, was confirmed by using WinXCOM data. The results of the MC model validation are shown in Table 1. A difference of less than $2 \%$ between the obtained results from the MC model and WinXCOM data was found.

The results of $\mu$ values for ordinary concrete and NCs containing 10, 20 and $30 \mathrm{wt} \%$ of Os, Ir and Ba NPs using three photon energies in terms of density are presented in Figure 5. The decline in $\mu$ value is observed with increasing energy in all three types of concrete with all weight fractions. Also, with increasing density, the $\mu$ values of NCs have increased, but this process was not linear, and in the case of NC containing $\mathrm{Ba}$, an rise in $\mu$ value was observed despite its low density.

In Figure 6, the $\mu$ values in terms of beam energy were plotted for all samples. As the results showed, at constant energy, by increasing weight fraction, the $\mu$ value has enlarged in all NCs. For example, in the case of NC containing $30 \mathrm{wt} \%$ Os, the $\mu$ value was about $16.9 \%$ higher than the $10 \mathrm{wt} \%$, and for $\mathrm{NC}$ containing Ir, the difference was about $18.4 \%$ and for Ba was about $14.8 \%$ using $18 \mathrm{MV}$ radiation beam.

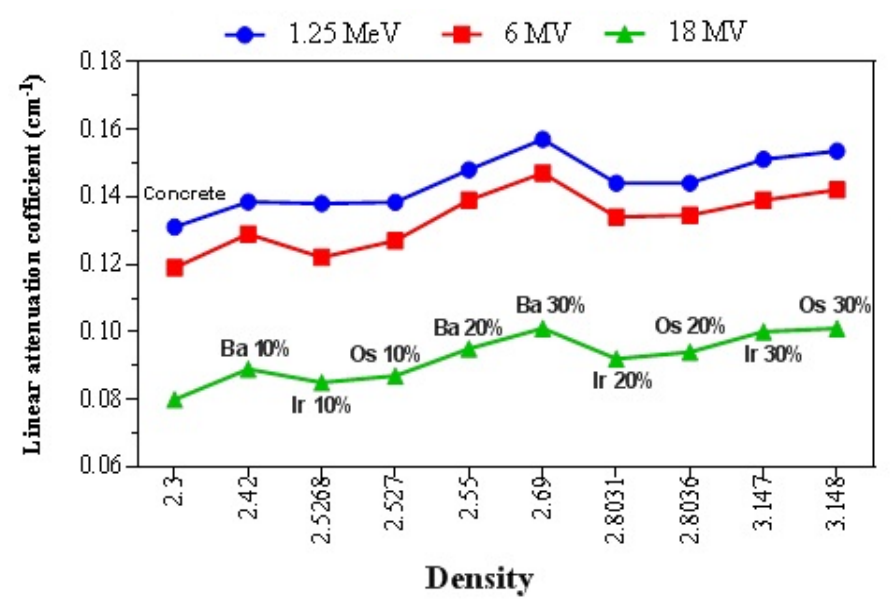

Figure 5. Linear attenuation coefficients $\left(\mathrm{cm}^{-1}\right)$ of high-density concretes for photon energy spectra of 6 and $18 \mathrm{MV}$ as well as ${ }^{60} \mathrm{Co}$ photons in terms of density.
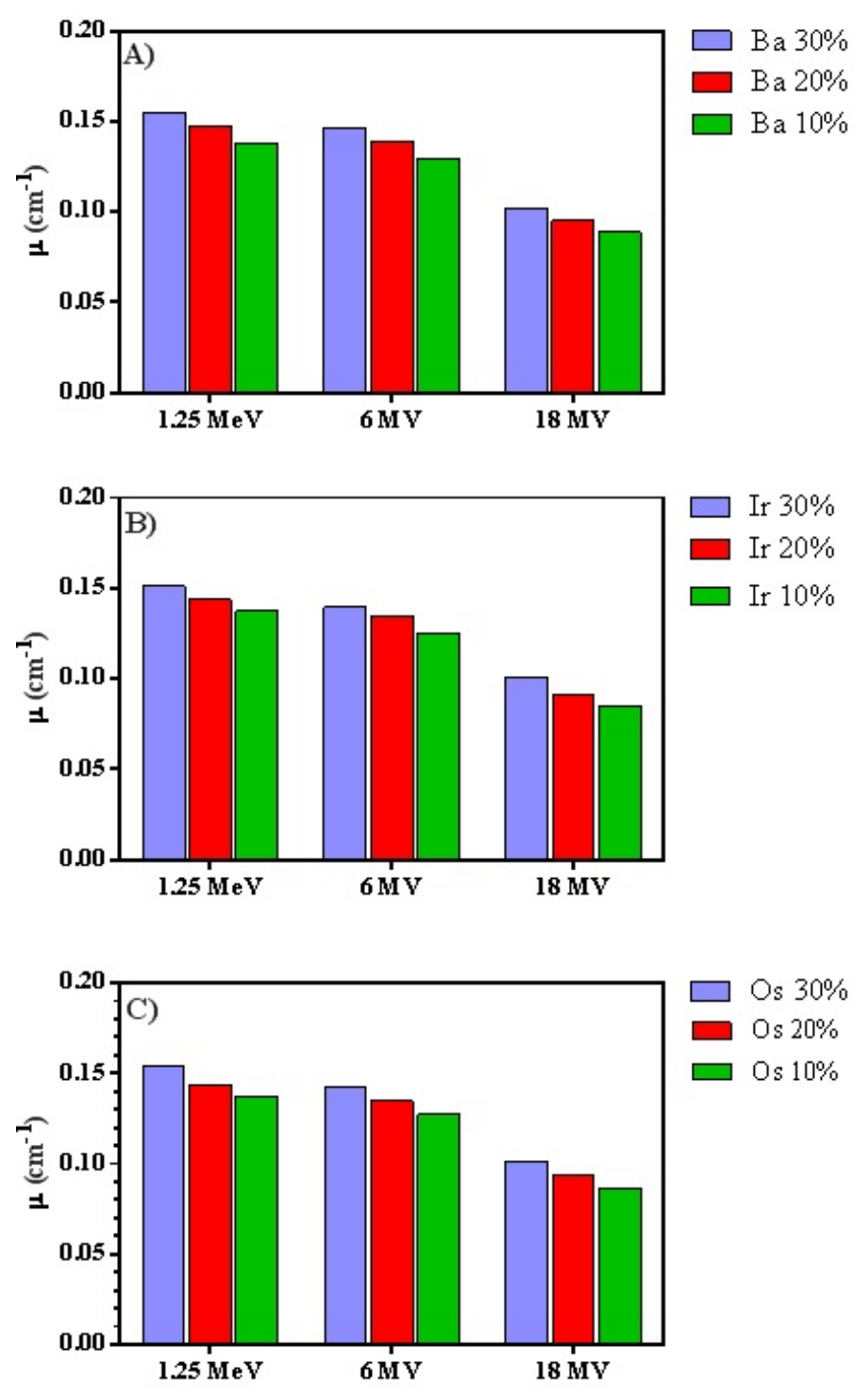

Figure 6. Linear attenuation coefficient values of (A) Nano Bariteconcrete (B) Nano Iridium-concrete $(C)$ Nano Osmium-concrete in different weight fractions, in terms of photon energy. 
Table 2. Composition, properties and linear attenuation coefficients of studied concretes for 6 and $18 \mathrm{MV}$ photon beam spectra as well as ${ }^{60} \mathrm{Co}$. NPs: Nanoparticles.

\begin{tabular}{|c|c|c|c|c|c|c|c|}
\hline & $\begin{array}{c}\text { Atomic } \\
\text { Composition } \\
\text { of NPs }\end{array}$ & $\begin{array}{l}\text { Electron Density of NPs } \\
\qquad\left\{N_{0}=\frac{N Z}{A}(e / g)\right\}\end{array}$ & $\begin{array}{l}\text { Weight } \\
\text { Fraction }\end{array}$ & $\begin{array}{r}\text { Density } \\
\left(\mathrm{g} / \mathrm{cm}^{3}\right)\end{array}$ & $\begin{array}{c}\text { Linear } \\
\text { Attenuation } \\
\text { Coefficient of } \\
{ }^{60} \mathrm{Co}\end{array}$ & $\begin{array}{c}\text { Linear } \\
\text { Attenuation } \\
\text { Coefficient of } \\
6 \mathrm{MV}\end{array}$ & $\begin{array}{c}\text { Linear } \\
\text { Attenuation } \\
\text { Coefficient of } \\
18 \mathrm{MV}\end{array}$ \\
\hline $\begin{array}{l}\text { Ordinary } \\
\text { concrete }\end{array}$ & - & - & - & 2.3 & 0.1306 & 0.1195 & 0.0798 \\
\hline \multirow{3}{*}{$\begin{array}{c}\text { Concrete } \\
+ \text { Osmium NPs }\end{array}$} & \multirow{3}{*}{${ }_{76}^{190} \mathrm{Os}$} & \multirow{3}{*}{$2.41 E 23$} & $10 \%$ & 2.527 & 0.1375 & 0.1273 & 0.0866 \\
\hline & & & $20 \%$ & 2.8036 & 0.1438 & 0.1347 & 0.0941 \\
\hline & & & $30 \%$ & 3.148 & 0.1535 & 0.1421 & 0.1012 \\
\hline \multirow{3}{*}{$\begin{array}{c}\text { Concrete } \\
+ \text { Iridium NPs }\end{array}$} & \multirow{3}{*}{${ }_{77}^{193} \mathrm{Ir}$} & \multirow{3}{*}{$2.4 E 23$} & $10 \%$ & 2.5268 & 0.1373 & 0.1247 & 0.085 \\
\hline & & & $20 \%$ & 2.8031 & 0.1436 & 0.1344 & 0.0916 \\
\hline & & & $30 \%$ & 3.147 & 0.1512 & 0.1394 & 0.1006 \\
\hline \multirow{3}{*}{$\begin{aligned} & \text { Concrete } \\
+ & \text { Barite NPs }\end{aligned}$} & \multirow{3}{*}{$\mathrm{BaSO}_{4}$} & ${ }_{56}^{138} \mathrm{Ba}=2.44 E 23$ & $10 \%$ & 2.42 & 0.138 & 0.1298 & 0.0887 \\
\hline & & ${ }_{16}^{32} S=3.011 E 23$ & $20 \%$ & 2.55 & 0.1477 & 0.1392 & 0.095 \\
\hline & & ${ }_{8}^{16} O=3.01 E 23$ & $30 \%$ & 2.69 & 0.1548 & 0.1462 & 0.1018 \\
\hline
\end{tabular}

The more details and results of the $\mu$ values for all types of concretes using the photon energy beam spectra of 6 and $18 \mathrm{MV}$ from the Varian Linac, as well as ${ }^{60} \mathrm{Co}$, with an average energy of 1.25 MeV is shown in Table 2 .

Figure 7 demonstrated the flux of scattered photons on a concrete wall with a thickness of $250 \mathrm{~cm}$, from an angle of 90 to $180^{\circ}$ with $10^{\circ}$ intervals for the photon beam energy of 6 and $18 \mathrm{MV}$ Varian Linac. In general, with the increasing the effective atomic number of concrete $\left(Z_{\text {eff }}\right)$, the flux of scattered photons per initial photon has increased at all angles. Also, the scattered photon flux for all types of concrete varied significantly at different angles.
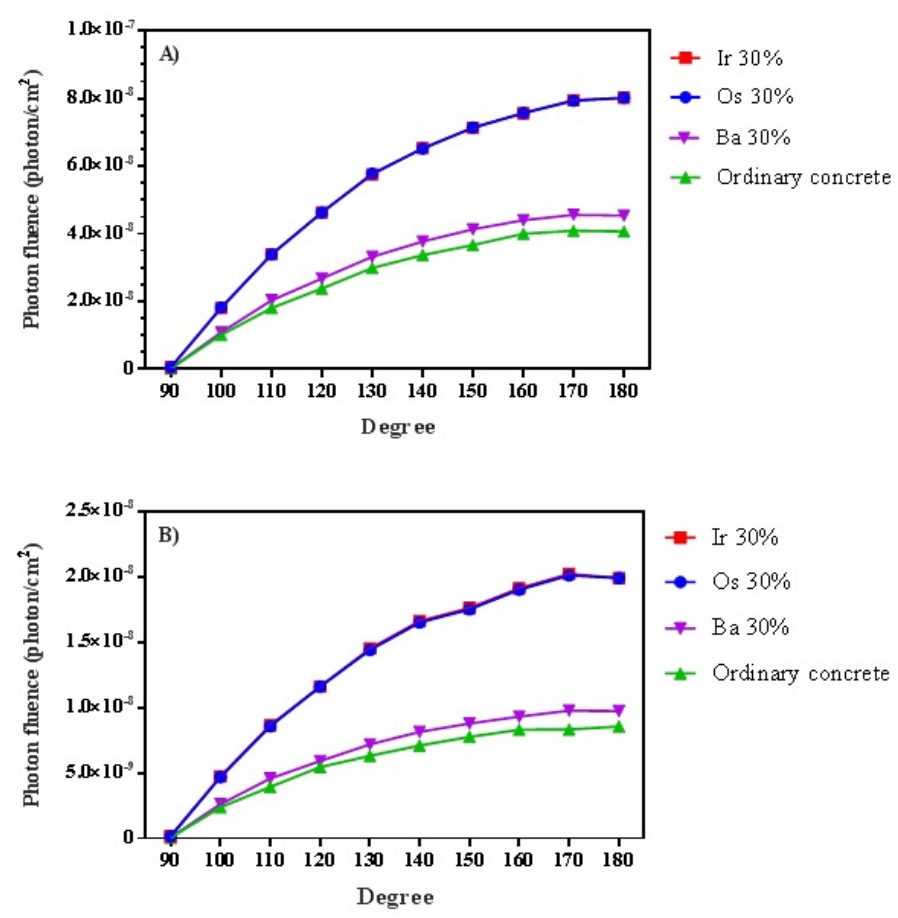

Figure 7. Scattered photon flux of studied Nano-concretes and ordinary concrete for A) $18 \mathrm{MV}$ and B) $6 \mathrm{MV}$ photon beam in terms of different degrees.

\section{Discussion}

For all samples of NCs as well as ordinary concrete, with increasing beam energy, the $\mu$ values decreased (Figure 5). Because at these ranges of beam energies, the Compton scattering is the dominant interaction between photons and materials, the probability of a Compton interaction is reduced by photon energy increasing for all elements. On the other hand, as the photon energy increases, the photon scattering at smaller angles is more likely to occur, and most of these events accrued in the forward direction. Because by increasing radiation energy, less energy is transferred to the scattered electron while most of the initial energy remains by the scattered photon. Overall, as a result, with increasing energy, the attenuation power of the shielding materials decreases and leads to a decrease in the $\mu$ value. $^{25}$ Also, as the density rises, the $\mu$ value increases (Figure 5) But not in linear manner. In the case of NCs containing Ir and Os, due to its high density of Os, it has shown higher $\mu$ values at all energies than NCs containing Ir. In the study by Waly et al., the simulation results showed that the HVL decreases in the energy of the photons from 0.1 to $15 \mathrm{MV}$ for different densities of concrete with increasing density. This means that a $50 \%$ increase in density provides a more than $50 \%$ reduction in $\mathrm{HVL}^{26}$ Also, the design of a radiotherapy bunker with concrete containing high density NPs has shown that the thickness required for radiation shielding been reduced by $0.8 \%$ compared to before. ${ }^{27}$

Despite the lower density of NCs containing Ba, it was found a slightly higher shielding properties than NCs containing Os and Ir with the same weight fraction (Figure 6). For example, at the same weight percentage $\left(30 \mathrm{wt} \%\right.$ ) and using ${ }^{60} \mathrm{Co}$ radiation beam, the $\mu$ amount of $\mathrm{NC}$ containing $\mathrm{Ba}$ is $0.85 \%$ and $2.4 \%$ higher than Os and Ir, respectively (Table 2). These differences for the photon beam of $18 \mathrm{MV}$ are $0.59 \%$ and $1.2 \%$ and for the photon beam of $6 \mathrm{MV}$, they reached $2.9 \%$ and $4.9 \%$, respectively. A parameter that can be considered involved in this increase could be the electron density of the fillers that make up NCs, the values of which are listed in Table 2. 
Regarding the energies used in radiotherapy, 1-20 MV, Compton scattering is more important than other interactions. Since free electrons (unbound) are involved in Compton scattering, where in this phenomenon, electron density plays an important role in the amount of radiation absorption. The electron densities for the Barite-forming atoms, $\mathrm{Ba}, \mathrm{S}$, and $\mathrm{O}$ are about $1.7 \%, 25.5 \%$, and $25.4 \%$ higher than those of the Os and Ir atoms, respectively. Due to the presence of these atoms in the form of NPs inside the concrete, and the increase in surface to volume ratio, the effect of this parameter on the attenuation of photons is intensified by the Compton phenomenon. This factor upsurges the Compton interaction cross-section in $\mathrm{Ba}$ containing $\mathrm{NC}$, which leads to an increase in beam attenuation and linear attenuation coefficient in these NCs. The effective atomic number, $Z_{\text {eff, }}$, and the effective electron number, $N_{\text {eff, }}$, have been shown to be useful parameters for the beam shield. $Z_{\text {eff }}$ and $N_{\text {eff }}$ depend on the main constituents of heavy concrete and ordinary concrete. According to the analyzed results, the concentrations of Iron, Barite, and Calcium in concrete are more effective than the concentrations of other materials. $Z_{\text {eff }}$ and $N_{\text {eff }}$ values in heavy concretes are higher than ordinary concretes. ${ }^{\mathbf{2 8}}$

For all samples of NCs containing $\mathrm{Ba}$, Os and Ir, with increasing the wt $\%$ of NPs in the concrete, the $\mu$ values at all three energies have increased (Figure 6). One of the reasons may be that the addition of Nano-material reduces the cement content by partially displacing the cement, based on weight, by increasing the conjunctions. ${ }^{29}$ As the weight percentage increases, this diminishes the pores and increases the concrete density. Therefore, by increasing the wt $\%$, the $\mu$ value rises. In the study of Jankovic et al., a heavy concrete with Nano-Silica and a combination of $\mathrm{Ba}$ and Quartz was investigated. This composite showed fine pore size distribution, improved compressive strength and suitable radiation protective properties for the energy range from $10 \mathrm{keV}$ to $150 \mathrm{MeV} .^{29}$

The flux of scattered photons of all concrete samples at angles of $90^{\circ}$ to $180^{\circ}$ for the 6 and $18 \mathrm{MV}$ energy photon spectra of Varian Linac is presented in Figure 7. The highest scattered flux is related to NC containing $30 \%$ Os, and the lowest scattered flux at all angles is related to ordinary concrete. The composition of concrete components affects the photon scattering flux. Because the interaction of pair production with material, increasing with their atomic number. As the atomic number grows, the electric field of the nucleus also strengthens. The absorption coefficient of pair production for each atom depends on the square of the atomic number $\left(Z^{2}\right)$. By increasing the interaction of pair production, the production of annihilation particles also increases due to the annihilation phenomenon, which will result in a higher photon scattering flux in concretes containing particles with higher atomic numbers. ${ }^{28}$ Another point that can be deduced from the Figure is that the scattered photon flux for all types of concrete varies considerably at different angles. In fact, the photon scattering flux at larger angles is more likely to occur for all types of concrete. For example, in the case of NC containing Os, the photon flux at $180^{\circ}$ is approximately 4.4 times higher than the photon flux at $100^{\circ}$ (Figure 7). Because at angles close to 180 degrees, there is less material in the scattered radiation path to penetrate, therefore, due to the less absorbent material in the scattered radiation path, the scattered beam flux at angles close to 180 degrees is higher than the lateral angles. ${ }^{\mathbf{3 0}}$ The flux ratio of the scattered photons for the photon beam energy spectra of $6 \mathrm{MV}$ at each angle is somewhat similar to that of an 18MV photon beam (Figure 7). For ordinary concrete, the change in scattered photon flux with respect to the angle occurs for the $18 \mathrm{MV}$ photon beam energy with a steeper slope than the $6 \mathrm{MV}$ photon beam. Also, in all samples, the flux of scattered beams for photons of $18 \mathrm{MV}$ is higher than $6 \mathrm{MV}$. In fact, increasing the energy leads to an increase in the backscatter dose rate, because the dose rate is directly proportional to the initial energy. Also, the probability of producing a particle of annihilation resulting from the production of a pair production growths with increasing energy. For energies higher than $7 \mathrm{MV}$, these photons of annihilation are more likely to be produced. ${ }^{31}$ Similarly, the ordinary concrete had the lowest scattered photon flux, and the highest scattered photon flux was for $\mathrm{NC}$ containing $30 \%$ Os at $6 \mathrm{MV}$ photon beam. This can be attributed to the high atomic number of Os.

In the used energy range, Compton scattering and pair production are the main scattering factors. In the present study, the macroscopic behaviour of scattered photons by heavy NC has been shown that the presence of atoms with a high number of atoms has significantly increased the flux of scattered photons in all directions. In the studied concretes, the intensity of photon scattering occurred at an angle of 180 degrees more than 90 degrees. Despite all novelties and high workload, further experimental studies are recommended for validation of our MC study.

Another point that should be noticed is the application of a homogenous and orderly arrangement of nanoparticles inside the concrete matrix in the current study. In other words, in such a lined-up structure, the impact of overlapping is more pronounced than it can be seen in reality, and consequently, the MC results may vary slightly from the measurements. The main reason for this type of modelling originates from the fact that it was not possible to model the random distribution of nanomaterials inside concrete with MCNPX code and simplistically, the pattern with the overlapping effect of nanoparticles in the pathway of the photon beam was modelled.

\section{Conclusion}

The linear attenuation coefficients and the photon scattering fluxes of three types of heavy Nano-concrete at different weight percentages were calculated using the 6 and $18 \mathrm{MV}$ photon beam spectrum of Varian Linac, as well ${ }^{60} \mathrm{Co}$ with an average beam energy of $1.25 \mathrm{MeV}$.

The effect of density, atomic number, electron density and photon beam energy was investigated. It was observed that the addition of Iridium, Osmium and Barite NPs to concrete led to 
an increase in linear attenuation coefficient compared to ordinary concrete due to their high density. Also, concrete containing Barite NPs, despite having a low density compared to Nano-concrete containing Iridium and Osmium, showed a higher linear attenuation coefficient due to its high electron density atoms. The results revealed the dependence of the photon scattering flux on the effective atomic number of concrete. In other words, scattering flux increased with a rising atomic number of filler NPs.

The shielding properties of Iridium and Osmium NPs were investigated for the first time in the current study, which showed that they have slightly lower linear attenuation coefficients relative to Barite Nano-concretes while they exhibited higher photon scattering flux than Barite-containing Nano-concretes.
Finally, the authors believe that the results of the current MC study should be investigated and confirmed with more experimental works.

\section{Acknowledgement}

The authors would like to thank Medical Radiation Science Research Team of Tabriz University of Medical Sciences for financial support under MSc thesis in Medical Physics. (Ethical code: IR.TBZMED.VCR.REC.1399.219.)

\section{Conflict of interest}

The authors declare that there is no conflict of interest.

\section{References}

1. Tekin H, Sayyed M, Issa SA. Gamma radiation shielding properties of the hematite-serpentine concrete blended with $\mathrm{WO}_{3}$ and $\mathrm{Bi}_{2} \mathrm{O}_{3}$ micro and nano particles using MCNPX code. Radiation Physics and Chemistry. 2018;150:95-100. https://doi.org/10.1016/j.radphyschem.2018.05.002

2. Janković K, Stanković S, Bojović D, Stojanović M, Antić L. The influence of nano-silica and barite aggregate on properties of ultra high performance concrete. Construction and Building Materials. 2016;126:147-156. https://doi.org/10.1016/j.conbuildmat.2016.09.026

3. Mesbahi A, Mansouri E, Jangjoo AG, Tekin HO. Radiation protection characteristics of nano-concretes against photon and neutron beams. Smart Nanoconcretes and Cement-Based Materials: Elsevier; 2020:447-460. https://doi.org/10.1016/B978-0-12-8178546.00019-2

4. Malekzadeh R, Mehnati P, Sooteh MY, Mesbahi A. Influence of the size of nano-and microparticles and photon energy on mass attenuation coefficients of bismuth-silicon shields in diagnostic radiology. Radiological Physics and Technology. 2019;12(3):325-334. https://doi.org/10.1007/s12194-019-00529-3

5. Zabihzadeh M, Ay MR, Allahverdi M, Mesbahi A, Mahdavi SR, Shahriari M. Monte Carlo estimation of photoneutrons contamination from high-energy X-ray medical accelerators in treatment room and maze: a simplified model. Radiation Protection Dosimetry. 2009;135(1):21-32. https://doi.org/10.1093/rpd/ncp097

6. Juste B, Morató S, García C, Miró R, Verdú G. Monte Carlo code application to the study of 3D neutrons distribution in a radiotherapy bunker and validation with experimental measurements. Nuclear Instruments and Methods in Physics Research Section A: Accelerators, Spectrometers, Detectors and Associated Equipment. 2020;954:161248. https://doi.org/10.1016/j.nima.2018.09.083

7. Khaldari R, Mesbahi A, Kara U. Monte Carlo calculation of shielding properties of newly developed heavy concretes for megavoltage photon beam spectra used in radiation therapy. Iranian Journal of Medical Physics. 2016;13(4):250-260. https://dx.doi.org/10.22038/ijmp.2017.19206.1175

8. Ahmad I, Shahzada K, Ahmad MI, et al. Densification of Concrete using Barite as Fine Aggregate and its Effect on Concrete Mechanical and Radiation Shielding Properties. Journal of Engineering Research. 2019;7(4):81-95.

9. Mortazavi S, Mosleh-Shirazi M, Roshan-Shomal P, Raadpey N, Baradaran-Ghahfarokhi M. High-performance heavy concrete as a multi-purpose shield. Radiation Protection Dosimetry. 2010;142(2-4):120-124. https://doi.org/10.1093/rpd/ncq265

10. Tekin H, Sayyed M, Altunsoy E, Manici T. Shielding properties and effects of $\mathrm{WO}_{3}$ and $\mathrm{PbO}$ on mass attenuation coefficients by using MCNPX code. Dig. J. Nanomater. Biostruct. 2017;12(3):861-867.

11. Agar O, Tekin HO, Sayyed M, Korkmaz ME, Culfa O, Ertugay C. Experimental investigation of photon attenuation behaviors for concretes including natural perlite mineral. Results in Physics. 2019;12:237-243. https://doi.org/10.1016/j.rinp.2018.11.053

12. Rajavikraman R. Novel method for radiation shielding using nano-concrete composite. Int J Mater Sci Eng. 2013;1:20-23. https://doi.org/10.12720/ijmse.1.1.20-23

13. Krishna BG, Prasad P, Sahu V, Sahu JP, Agarwal A. Beta Backscattering and Gamma Radiation Absorption Characteristics of Carbon Nanoparticles Contained Concrete Composite. Paper presented at: Nano Hybrids and Composites 2017. https://doi.org/10.4028/www.scientific.net/NHC.17.31

14. Tekin HO, Singh VP, Manici T. Effects of micro-sized and nano-sized $\mathrm{WO}_{3}$ on mass attenauation coefficients of concrete by using MCNPX code. Applied Radiation and Isotopes. 2017;121:122-125. https://doi.org/10.1016/j.apradiso.2016.12.040 
15. Verdipoor K, Alemi A, Mesbahi A. Photon mass attenuation coefficients of a silicon resin loaded with $\mathrm{WO}_{3}, \mathrm{PbO}$, and $\mathrm{Bi}_{2} \mathrm{O}_{3} \mathrm{Micro}$ and Nano-particles for radiation shielding. Radiation Physics and Chemistry. 2018;147:85-90. https://doi.org/10.1016/j.radphyschem.2018.02.017

16. Facure A, Silva A, Rivera J, Falcao R. Neutron scattering in concrete and wood: Part II-oblique incidence. Radiation Protection Dosimetry. 2008;128(3):367-374. https://doi.org/10.1093/rpd/ncm378

17. Abdo AE-S. Calculation of the cross-sections for fast neutrons and gamma-rays in concrete shields. Annals of Nuclear Energy. 2002;29(16):1977-1988. https://doi.org/10.1016/S0306-4549(02)00019-1

18. Mesbahi A, Azarpeyvand A-A, Shirazi A. Photoneutron production and backscattering in high density concretes used for radiation therapy shielding. Annals of Nuclear Energy. 2011;38(12):2752-2756. https://doi.org/10.1016/j.anucene.2011.08.023

19. Mesbahi A, Azarpeyvand A-A, Khosravi HR. Does concrete composition affect photoneutron production inside radiation therapy bunkers? Japanese Journal of Radiology. 2012;30(2):162-166. https://doi.org/10.1007/s11604-011-0030-y

20. Choi CH, Park S-Y, Park JM, Chun M, Kim J-i. Monte Carlo simulation of neutron dose equivalent by photoneutron production inside the primary barriers of a radiotherapy vault. Physica Medica. 2018;48:1-5. https://doi.org/10.1016/j.ejmp.2018.03.009

21. Mesbahi A, Alizadeh G, Seyed-Oskoee G, Azarpeyvand A-A. A new barite-colemanite concrete with lower neutron production in radiation therapy bunkers. Annals of Nuclear Energy. 2013;51:107-111. https://doi.org/10.1016/j.anucene.2012.07.039

22. Mesbahi A, Khaldari R. Neutron and photon scattering properties of high density concretes used in radiation therapy facilities: A Monte Carlo study. Polish Journal of Medical Physics and Engineering. 2017;23(3):61. https://doi.org/10.1515/pjmpe-2017-0011

23. Pelowitz DB. MCNPX USER'S MANUAL Version 2.7. 0-LA-CP-11-00438. Los Alamos National Laboratory. 2011.

24. Sheikh-Bagheri D, Rogers D. Monte Carlo calculation of nine megavoltage photon beam spectra using the BEAM code. Medical Physics. 2002;29(3):391-402. https://doi.org/10.1118/1.1445413

25. Mansouri E, Mesbahi A, Malekzadeh R, Mansouri A. Shielding characteristics of nanocomposites for protection against X-and gamma rays in medical applications: effect of particle size, photon energy and nanoparticle concentration. Radiation and Environmental Biophysics. 2020:1-18. https://doi.org/10.1007/s00411-020-00865-8

26. Waly E-SA, Bourham MA. Comparative study of different concrete composition as gamma-ray shielding materials. Annals of Nuclear Energy. 2015;85:306-310. https://doi.org/10.1016/j.anucene.2015.05.011

27. Ghasemi-Jangjoo A, Ghiasi H. MC safe bunker designing for an $18 \mathrm{MV}$ linac with nanoparticles included primary barriers and effect of the nanoparticles on the shielding aspects. Reports of Practical Oncology \& Radiotherapy. 2019;24(4):363-368. https://doi.org/10.1016/j.rpor.2019.05.009

28. Un A, Demir F. Determination of mass attenuation coefficients, effective atomic numbers and effective electron numbers for heavyweight and normal-weight concretes. Applied Radiation and Isotopes. 2013;80:73-77. https://doi.org/10.1016/j.apradiso.2013.06.015

29. Norhasri MM, Hamidah M, Fadzil AM. Applications of using nano material in concrete: A review. Construction and Building Materials. 2017;133:91-97. https://doi.org/10.1016/j.conbuildmat.2016.12.005

30. Swanson WP. Radiological safety aspects of the operation of electron linear accelerators. 1979.

31. Raso DJ. Monte Carlo calculations on the reflection and transmission of scattered gamma rays. Nuclear Science and Engineering. 1963;17(3):411-418. https://doi.org/10.13182/NSE63-A17390 